\title{
FIRST RESPONDERS FIELD TRIALS OF SALIANT TECHNOLOGY
}

SALIANT - Selective Antibodies Limited Immuno Assay Novel Technology is the title of the research project developed and realised by a European consortium of biotechnology companies, universities and government forensic laboratories within the Seventh Framework Programme (FP7), Security Theme. The aim of this project was to develop a hand-held device for real-time analysis of trace levels of explosives, chemicals and drugs. The key innovation was a positive detection lateral-flow test for small molecules that is rapid, highly sensitive and simple to use making it ideally suited to deployment by First Responders and Forensic Service Providers at crime scenes and terrorist incidents. The Faculty of Special Engineering of the University of Zilina (FSE) as a project team member, was responsible for the task of evaluating the efficacy and sensitivity of the SALIANT technology for explosives detection for emergency responders' use. This paper provides the results and details about conducted experiments for the TNT explosive which were carried out in real-world conditions. The trials were specifically oriented on verification of the explosion products coverage and possibilities of their measuring by the SALIANT system.

Keywords: Security, explosive, first responder, field trials, SALIANT.

\section{Introduction}

Preservation and development of values of justice, freedom, and security is one the European Union main objectives. Although the European countries live in relative security, in their daily life they face many complex security threats and challenges. The fight against terrorism and organised crime, the protection of the external European borders and civil crisis management has gained the main importance. Four of five Europeans want more actions at EU level against organised crime and terrorism [1 and 2]. But no single Member state is able to respond to these threats on its own.

In February 2004, the European Commission launched a "Preparatory Action in the field of Security Research" (PASR) endowed with an estimated budget of $65 \mathrm{M} €$ for the period 2004-2006.. It was an important first step in addressing the need for Community action and aimed at establishing a fully-fledged Programme for Security Research in Europe from 2007. The Preparatory Action prepared the groundwork for a successful Security Research Programme [3 and 4].

The PASR was complemented by a number of projects funded under the 6th Framework Programme (FP6). In September 2004, the European Commission proposed the establishment of a "European Security Research Programme" (ESRP), to be funded over the period 2007-2013 under the $7^{\text {th }}$ Framework
Programme (FP7), endowed with an envisaged budget of 1.4 Bn€ [5].

Within the FP7, priority Security, the Faculty of Special Engineering of the University of Zilina (FSE) participated in the research project Selective Antibodies Limited Immuno Assay Novel Technology - SALIANT (2010-2013).

The SALIANT project was developed by a European consortium of biotechnology companies, universities and government forensic laboratories.

SALIANT aimed to develop a hand-held device for real-time analysis of trace levels of explosives, chemicals and drugs. The key innovation was a positive detection lateral-flow test for small molecules that is rapid, highly sensitive and simple to use making it ideally suited to deployment by First Responders and Forensic Service Providers at crime scenes and terrorist incidents.

Lateral flow immunodiagnostics has long offered the promise of fast, high quality testing for substances of low molecular weight such as explosives. There have, however, been very real challenges to bringing the full power of such technology to bear in this area. The problem is simply size. Large analytes can support the simultaneous binding of both capture and detector antibodies, allowing typical excess-reagent sandwich immunoassays to be formatted in which increasing analyte concentration provides an increase of observable signal over a very low zero background. Small molecules are simply not large enough to support such

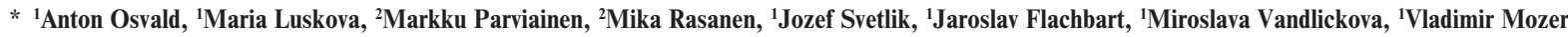
${ }^{1}$ Department of Fire Engineering, Faculty of Security Engineering, University of Zilina, Slovakia

${ }^{2}$ Reagena Oy Ltd, Toivala,, Finland

E-mail: anton.osvald@fbi.uniza.sk
} 
simultaneous binding. Alternative systems in effect measure how much analyte is not present. This brings major problems in terms of precision, sensitivity and read-out where, classically, increasing concentration of analyte reduces the signal produced, making point-of-need devices often difficult to read. What is required is a robust system in which there is no observable signal in the absence of analyte, and even low level samples give an obvious observable signal over this zero background.

SALIANT offers a system based on a small bindable molety that is first conjugated close to the binding site of a primary antibody against the analyte such that when analyte binds the antibody, the molety can still be bound by a labelled secondary antibody. A large reagent-analogue of the analyte is also introduced, binding analyte - unbound primary antibody, and thereby blocking binding of the secondary antibody to the molety. Thus the more analyte that is present the more binding of secondary antibody occurs and the more signal is produced [6].

\section{Scope, objectives and the method of work}

At present, taking and analysis of post-blast samples is carried out using standard methods. These methods use physical and chemical properties of the substances used in the manufacture of explosives. Sampling and subsequent analysis is carried out by laboratory methods IMS and LC MS that provide relevant results but they are demanding regarding to instrumentation and time. Method SALIANT focuses on sampling and detection of explosives based on the principle of immune assay.

The aim of the field trials was to verify the efficacy of the immunological method SALIANT for measuring explosion products.

\subsection{TNT - 2, 4, 6 - trinitrotoluene}<smiles>Cc1c([N+](=O)[O-])cc([N+](=O)[O-])cc1[N+](=O)[O-]</smiles>

Fig. 1 Chemical formula for TNT

It is yellow-coloured solid best known as a useful explosive material with convenient handling properties. The explosive property of TNT is considered to be the standard gauge of strength of other explosives. By detonation, TNT decomposes in these ways: $2 \mathrm{C}_{7} \mathrm{H}_{5} \mathrm{~N}_{3} \mathrm{O}_{6} \rightarrow 3 \mathrm{~N}_{2}+5 \mathrm{H}_{2} \mathrm{O}+7 \mathrm{CO}+7 \mathrm{C}$

$2 \mathrm{C}_{7} \mathrm{H}_{5} \mathrm{~N}_{3} \mathrm{O}_{6} \rightarrow 3 \mathrm{~N}_{2}+5 \mathrm{H}_{2}+12 \mathrm{CO}+2 \mathrm{C}$
Because TNT has an excess of carbon, explosive mixtures with oxygen-rich compounds can yield more energy per kilogram than TNT alone [7].

TNT is reported to contain 2.8 mega joules per kilogram explosive energy. The actual heat of combustion is 14.5 megajoules per kilogram, which requires that some of the carbon in TNT react with atmospheric oxygen, which does not occur in the initial event [8].

Quantitative methods used untill now are time consuming to evaluate what makes the investigation work more difficult. On the contrary, applied qualitative methods can quickly identify the various typical components of explosives but on the other hand the investigator can hardly map the whole area of events according to them.

Unlike the used methods, the system can also quantify the amount (concentration) in taken sample and thus to contribute to better and rapid mapping of the area of events after the explosion whereby the whole process of collection and analysis does not take more than 5 minutes.

\subsection{Field tests - experiment SALIANT}

\subsubsection{Conditions and course of the SALIANT experiment}

Based on the results of the zero experiment, the scheme of the sampling points for the experiment was adjusted (see Fig. 2). This experiment was carried out on April 9, 2013 in Kamenna Poruba - Zilina in the same area. Distances of sampling points from the explosion epicentre were shortened.

To capture detonation particles ceramic tiles, placed on the ground, were used. The samples were taken in direction of the axes 1, 2 and 3 in sequence from the explosion epicentre to the edge of the monitored area.

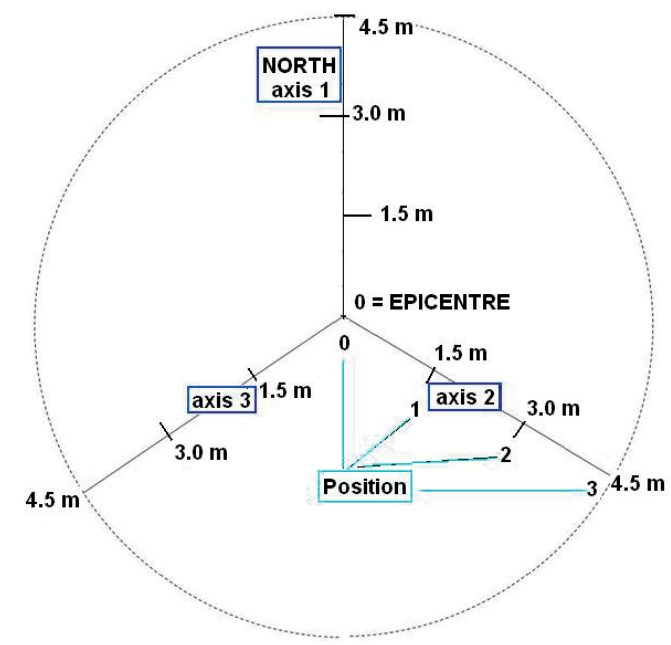

Fig. 2 Sampling points layout for the $1^{\text {st }}$ SALIANT experiment 
For explosions we used: 4 x $75 \mathrm{~g}$ of explosive (TNT technical quality, volume of TNT $\min .85 \%),(\mathrm{T})$.

\subsubsection{Course of the first experiment}

Figure 3 shows the experiment conditions. There was snow (several $\mathrm{cm}$ ). Meteorological situation in the time of explosions is given in Table 1. Figure 4 shows collection of explosion products by SALIANT method.

Meteorological situation during explosions - first experiment Table 1

\begin{tabular}{|c|c|c|c|c|c|c|c|}
\hline 节 & 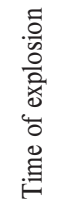 & 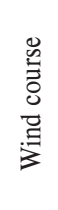 & 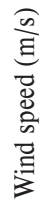 & 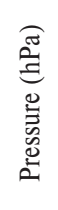 & 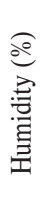 & 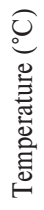 & $\frac{\text { 음 }}{\frac{0}{0}}$ \\
\hline 9. 4. 2013 & $8: 45$ & NE & 0.7 & 980 & 68 & 1.7 & TNT 1 \\
\hline 9. 4. 2014 & $9: 53$ & ES & 0.4 & 976 & 75 & 2.6 & TNT 2 \\
\hline
\end{tabular}

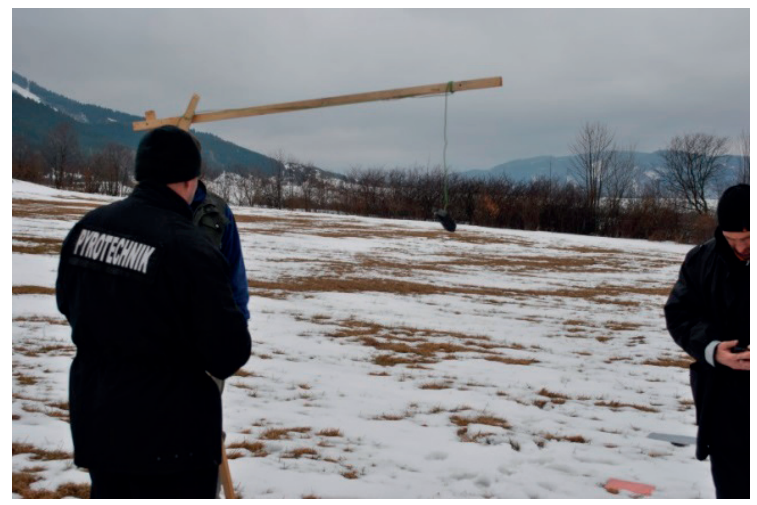

Fig. 3 Position of explosive charge and meteorological situation before explosion

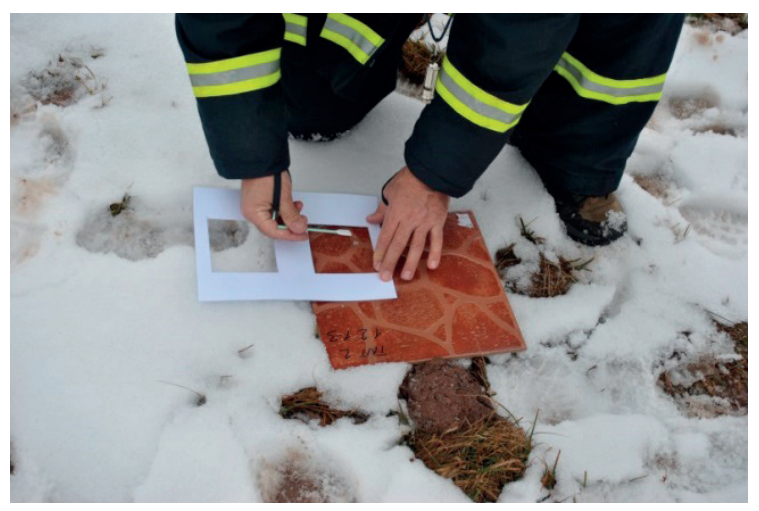

Fig. 4 SALIANT wet wipe

\subsubsection{Collection and evaluation of post blast products by SALIANT method}

SALIANT method requires special tools to collect explosion products (see Fig. 5) as well as special process for chemical analytical evaluation of the sample (see Fig. 6).

\section{Materials}

- MilliQ water,

- Methanol,

- Glass tube 130 x $16 \mathrm{~mm}$,

- Polyester TX715 swab (Basan),

- Paper mould for a square $10 \times 10 \mathrm{~cm}$.

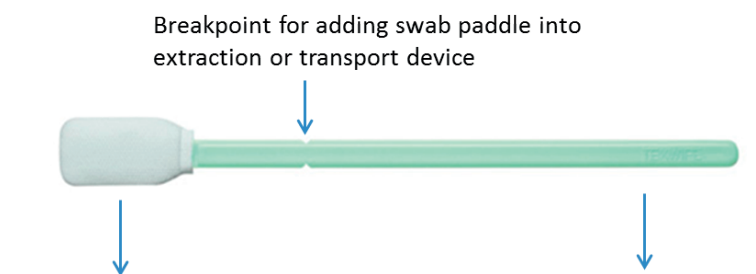

Rigid paddle
Double layer polyester
Thermally sealed

Polypropylene handle

Fig. 5 Polyester TX715 swab
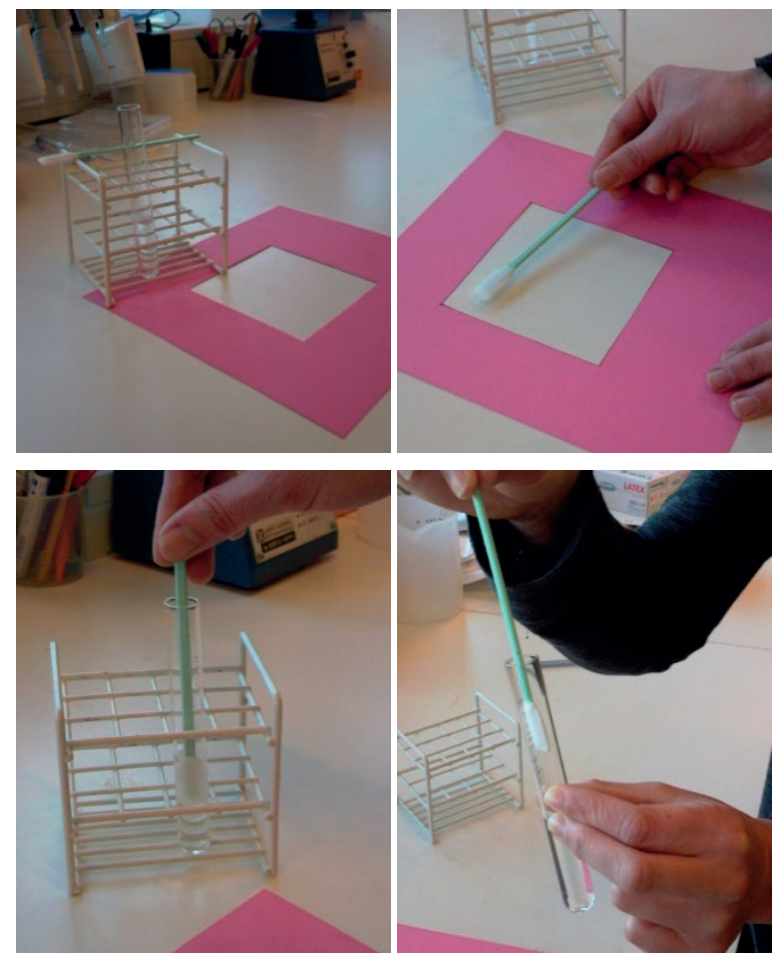

Fig. 6 Sampling of surfaces. Materials needed for wipe surface sampling (a), wiping a designated area with a swab (b), adding the swab to a tube with $5 \mathrm{ml}$ methanol (c) and removal of swab from extract after incubation (d). 
Sampling Method

1. Pre-wet swab with MilliQ water. Approximately $500 \mu \mathrm{l} / \mathrm{swab}$, no dripping of liquid from swab.

2. Wipe area of $10 \times 10 \mathrm{~cm}$, use the paper pattern for the correct area - see Fig. 2.

Extraction with methanol:

- Add $5 \mathrm{ml}$ of methanol to a clean glass tube.

- Place the swab in the tube.

- Shake and let it stand for 15 minutes at room temperature.

- Remove swab from tube.

- Homogenise the extract and use part of the extract for immunoassay.

- Store extract at $4{ }^{\circ} \mathrm{C}$ for conformation with LC/MS.

\section{Dilute extract with LFIA assay buffer:}

Dilute the sample $1 / 10$ in LFD running buffer $(100 \mathrm{mM}$ PBS with $0.25 \%$ BSA, $0.1 \%$ Triton X 100) NB final loading of Methanol should not exceed $10 \%$.

\section{Proceed with protocol immunoassay SALIANT}

Add $80 \mathrm{ml}$ of the extracted sample dropwise into the small aperture of the LFD cassette. A clear band of gold should been seen at the bottom of the window shortly after this. If no clear band is seen LFD may not be running correctly and repetition will be necessary.

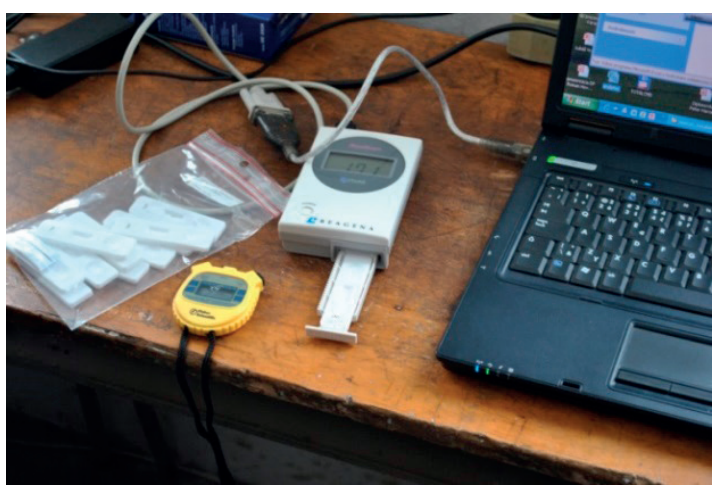

Fig. 7 SALIANT wet wipe of the TNT explosive

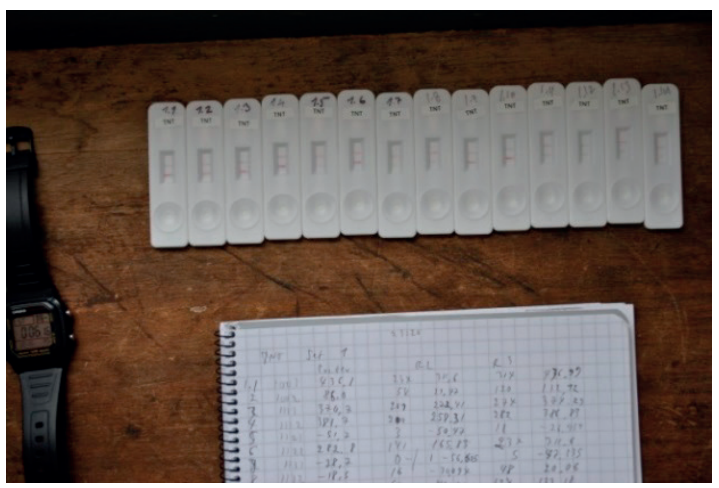

Fig. 8 Results
The wipes were evaluated by the reader (see Fig. 7). Figure 7 shows the reader with inserted wet strip and connected with computer. Figure 8 shows the results of experiment of April 9, 2013.

\section{Results}

The results of two explosions of each explosive are given in Tables 2 and 3. The first column indicates the No. of the sample with type of explosive (TNT - (T)). The second column indicates position at the explosion axis (A1, A2, A3) and distance from epicentre $(1.5 ; 3.0 ; 4.5 \mathrm{~m})$ (see Fig. 1), the third and fourth columns indicate measurements, results of the reader, the third column - data after $2^{\text {nd }}$ minute, the fourth column - data after $3^{\text {rd }}$ minute. Symbol BG indicates the background (purity before explosion), epicentre is the place vertically below charge. The values in the second and third minutes are different. Regarding the needs to develop high-speed detection system, it is assumed to make the methodology for measuring the second minute more accurate. For comparison the results, taken samples were analyzed also by current method IMS (Ionscan 4000) which did not indicate presence of the explosives in any of the samples.

The results of measurements by SALIANT

for the first TNT explosion

Table 2

\begin{tabular}{|c|c|c|c|}
\hline Code & Position & After 2nd min & After 3rd min \\
\cline { 3 - 4 } & & {$[\mathrm{ppb}]$} & {$[\mathrm{ppb}]$} \\
\hline $\mathrm{T} 1.0$ & $\mathrm{BG}$ & & \\
\hline $\mathrm{T} 1.1$ & epicentre & 234 & 314 \\
\hline $\mathrm{T} 1.2$ & $\mathrm{~A} 1 / 1.5$ & 54 & 120 \\
\hline $\mathrm{T} 1.3$ & $\mathrm{~A} 1 / 3.0$ & 209 & 274 \\
\hline $\mathrm{T} 1.4$ & $\mathrm{~A} 1 / 4.5$ & 200 & 282 \\
\hline $\mathrm{T} 1.5$ & $\mathrm{~A} 2 / 1.5$ & 3 & 18 \\
\hline $\mathrm{T} 1.6$ & $\mathrm{~A} 2 / 3.0$ & 141 & 234 \\
\hline $\mathrm{T} 1.7$ & $\mathrm{~A} 2 / 4.5$ & 1 & 5 \\
\hline $\mathrm{T} 1.8$ & $\mathrm{~A} 3 / 1.5$ & 16 & 48 \\
\hline $\mathrm{T} 1.9$ & $\mathrm{~A} 3 / 3.0$ & 62 & 124 \\
\hline $\mathrm{T} 1.10$ & $\mathrm{~A} 3 / 4.5$ & 171 & 271 \\
\hline
\end{tabular}

The experiment confirmed the quality and sensitivity of the explosion products measurements. The BG (background) values were null. Because this area is usually used for making various indicated experiments it was needed to carry out check measurements. The area was free of explosion traces left from previous explosions. It is reasonable that wind affects direction and quantity of explosion products. This fact was confirmed by our experiments that indicated sensitivity of measurement by SALIANT. It is documented also by the graphs (Figs. 9 and 10). 
The results of measurements by SALIANT for the second TNT explosion

Table 3

\begin{tabular}{|c|c|c|c|}
\hline \multirow{2}{*}{ Code } & Position & After 2nd min & After 3rd min \\
\cline { 3 - 4 } & & {$[\mathrm{ppb}]$} & {$[\mathrm{ppb}]$} \\
\hline T2.0 & BG & & \\
\hline T2.1 & epicentre & 11 & 43 \\
\hline T2.2 & A1 / 1.5 & 32 & 69 \\
\hline T2.3 & A1 / 3.0 & 5 & 53 \\
\hline T2.4 & A1 / 4.5 & 1 & 19 \\
\hline T2.5 & A2/1.5 & 31 & 92 \\
\hline T2.6 & A2/3.0 & 84 & 142 \\
\hline T2.7 & A2 / 4.5 & 77 & 137 \\
\hline T2.8 & A3/1.5 & 17 & 64 \\
\hline T2.9 & A3 / 3.0 & 31 & 81 \\
\hline T2.10 & A3 / 4.5 & 189 & 240 \\
\hline
\end{tabular}

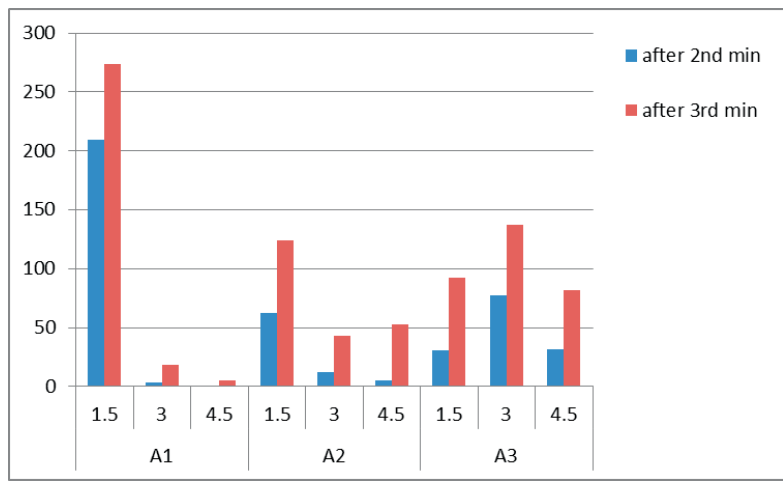

Fig. 9 First TNT explosion - results of measured values by SALIANT method

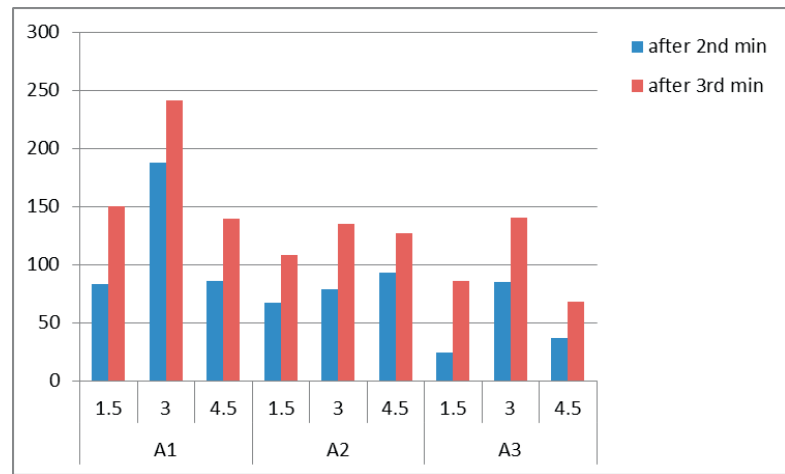

Fig. 10 Second TNT explosion - results of measured values by SALIANT method

The first experiment (Fig. 9) was performed under conditions of slow dead wind from the north side that blew away more explosion products within distance $1.5 \mathrm{~m}$ in axis A1. Minimum quantity fell in a distance of 4.5 and $3.0 \mathrm{~m}$. . On other axes (A2 and A3) the explosion products were distributed proportionally.

The second experiment (Fig. 10) was performed under conditions of slow wind of south direction, which resulted in higher concentration of explosion products on axis A1, especially in position 2 (three meters). Other positions had proportional arrangement of explosion products with regard to collection place and atmospheric conditions.

The SALIANT system allows detection of other groups of explosives (RDX , HMX) in addition to TNT detection.

\section{Conclusion}

The SALIANT project yielded many positive experiences and benefits for the University of Zilina team. The first major benefit was the work experience in an international team of experts. The task our team was responsible for also brought its own challenges. The goal was aimed at the identification of dangerous substances, explosives and drugs. The area of explosives, which became the major objective of the project, was a new experience for us and required significant amount of learning and research into the topic for the UNIZA project team. With the assistance of the partner organisations we were successfully able to devise the methodology for the field tests. This was also positively appraised at the project workshop that was held at the University of Zilina at which the field tests and their outcomes were presented.

The results of the field trials confirmed two basic hypotheses. Firstly, the dimensional parameters of field trials were validated for low amounts of explosives together with the ways of residual sample collection for forensic purposes. Secondly, the SALIANT methodology, based on the immunological identification of explosives residue, was validated, as well as its qualitative and quantitative sensitivity [9].

The experimental work was quite difficult. The field trials were carried out in an open terrain affected by adverse weather conditions. Heavy machinery was required to manipulate and place the test vehicles in the right location on site. This had to be combined with high laboratory precision and sensitive electronic analysis. Is should be noted that not only Stage 6 - Field trials, but the entire SALIANT project required dedicated and focused work from all involved teams.

\section{Acknowledgments}

The paper presents the results of the research, namely the field tests carried out in the project SALIANT. 


\section{References}

[1] Directorate - General for Internal Policies: Citizens Rights and Constitutional Affairs. Edition 5/2011[online]. [cited 1March 2012]. Available at: http://www.statewatch. org/news/2010/nov/ep-review-security-research-programme.pdf

[2] Research for a Secure Europe - Report of the Group of Personalities in the field of Security Research, Luxembourg : Office for Official Publications of the European Communities, 2004, ISBN 92-894-6611-1

[3] Selective Antibodies. 2013. Home. [online]. [cited 14 October 2013]. Available at: http://www.selectiveantibodies.com/

[4] BABRAUSKAS, VYTENIS: Ignition Handbook. Issaquah: WA: Fire Science Publishers/Society of Fire Protection Engineers, 2003, p. 453, ISBN 0-9728111-3-3.

[5] KlOUDA, K., KUBATOVA, H., ZEMANOVA, E.: Nanomaterials: Pros and Cons. Communications - Scientific Letters of the University of Zilina, 2011, vol. 13, pp. 6-12, ISSN 1335-4205

[6] JEANDESBOZ, J., RAGAZZI, F.: Review of Security Measures in the Research Framework Programme. European Parliament: Brussels, 2010 [online]. [cited 28 February 2012]. Available at: http://www. europarl.europa.eu/studie

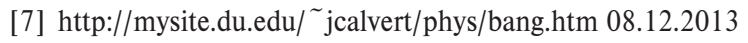

[8] TNS Opinion \& Social: Special Eurobarometer 371 Internal Security, Fieldwork: June 2011, Publication: November 2011. [online]. [cited 1March 2012]. Available at: http://ec.europa.eu/public_opinion/archives/ebs/ebs_371_en.pdf .

[9] OSVALD, A. et. al.: SALIANT - Selective Antibodies Limited Immuno Assay Novel Technology. Zilina : EDIS University of Zilina, p. 121, ISBN 978-80-554-0838-5. 